\title{
Review
}

\section{Regression Models with Stochastic Regressors: An Expository note}

\author{
Sulaxana Bharali ${ }^{*}$, Jiten Hazarika ${ }^{2}$ \\ 1 Department of Statistics, Dibrugarh University-4, Assam; sulaxana.bharali@gmail.com \\ 2 Department of Statistics, Dibrugarh University-4, Assam; jitenhazarika@rediffmail.com
}

\begin{abstract}
Regression models form the core of the discipline of econometrics. One of the basic assumptions of classical linear regression model is that the values of the explanatory variables are fixed in repeated sampling. However, in most of the real life cases, particularly in economics the assumption of fixed regressors is not always tenable. Under a non-experimental or uncontrolled environment, the dependent variable is often under the influence of explanatory variables that are stochastic in nature. There is a huge literature related to stochastic regressors in various aspects. In this paper, a historical perspective on some of the works related to stochastic regressor is being tried to pen down based on literature search.
\end{abstract}

Keywords: Non-normality, Classical Linear Regression Model, Modified Maximum Likelihood Estimation.

\section{Introduction}

In the realm of present day economic relationship and due to dynamic increase of demands of rapidly increasing population, econometric has become an important task in relatively every field. Econometric is the art of utilizing the factual techniques for the measurement of economic relations. An econometric study begins with a set of propositions about some characteristics of the economy. The theory specifies a set of precise, deterministic relationships among factors. An econometric model is a simplified representation of a real world process.

Regression models form the foundation of the discipline of econometrics. This class of model begins with a simple linear regression model. It is a statistical technique for investigating and modeling relationships between two variables i.e. the explained and the explanatory variable. It helps to estimate the strength and direction of the relationship, allowing predictions about past, present or future events to be made with information about past or present events. In broad sense, there are two types of linear regression models- General Linear Regression Models and Generalized Linear Regression Models. This classification is based on the distribution of the disturbance term. The usual notation of a regression model may be defined as-

$$
\widetilde{Y}=X \tilde{\beta}+\widetilde{e}
$$

where $\tilde{Y}$ is the vector of responses, $X$ is the data matrix and $\tilde{e}$ is the identically and independently distributed (iid) vector of disturbance terms or errors. $\tilde{\beta}$ is the vector of regression coefficient that represents the rate of change of one variable $(Y)$ as a function of changes in the other variable $(X)$. There are some prefixed assumptions, which the model must follow for the validity of the test. Among them, the design variable $X$ has traditionally been taken to be nonstochastic and the random error $e$ assumed to be normally distributed. 
The distribution of the random variable $e$ remains the same for all explanatory variables. If $e$ follows Normal distribution with mean 0 and variance $\sigma^{2}$, it is called Classical Linear Normal Regression Model. However, the usual assumption of normality and constant variance are not always satisfied. The alternative approach to this is called the Generalized Linear Regression Models where $e$ follows the distribution of the members of the exponential family of distribution, which includes Normal, Binomial, Poisson, Exponential, Gamma distribution, etc as its member. Also, the distribution of the random variable $e$ remains the same for all explanatory variables.

Another important assumption of regression model is explanatory variables are fixed in repeated samples. However, in many cases the assumption of non-stochastic regressor is not always tenable. This is valid for experimental work, in which the experimenter has control over the explanatory variables and can repeatedly observe the outcome of the dependent variable with the same fixed values or some designated values of the explanatory variables. In the social sciences and particularly in economics, explanatory variables in one equation are often generated as the outcome variables of other equations that are stochastic in nature. Thus they neither have the same fixed values in repeated samples nor do they have values that conform to the investigator's desired experimental design. Thus, under a non-experimental or uncontrolled environment, the dependent variable is often under the influence of explanatory variables that are stochastic in nature.

A problem of major importance is to estimate the parameters $\beta^{\prime}$ sand test the null hypothesis $H_{0}$ : $\beta_{i}=0$. Clearly, $H_{0}$ implies that the design variable has no effect on the response $Y$. Traditionally, assume that $e^{\prime} s(1 \leq i \leq n)$ are iid normal $N\left(0, \sigma^{2}\right)$. Various procedures have been developed for parameter estimation and inference in linear regression. Least Squares method is applied which give the best linear unbiased estimator when the basic assumptions were fulfilled. Since the method of maximum likelihood has all the desirable optimal properties under some general regularity conditions and the distribution of the error terms is known to belong to a certain parametric family of probability distributions, so we can employ this method also to estimate the parameters. The resulting estimate is identical to the OLS estimate when the distribution of the error term is normal. This work is devoted to historical perspective on stochastic regressions and its further developments.

\section{Regression Models with Stochastic Regressor}

Giving more emphasis on the assumption, that the design variable $X$ is traditionally been taken as nonstochastic, it is realized that the assumption of nonstochastic regressor is not always plausible, the regressor may be stochastic in nature. The term stochastic regressor means that the regressors, i.e. the explanatory variables are random with the change of time. The basic assumption in case of Stochastic regressors are: i) $X, Y, e$ random ii) $(X, Y)$ obtained from iid sampling iii) $\mathrm{E}(e \mid X)=0$ iv) $X$ takes atleast two values v) $\operatorname{Var}(e \mid X)=\sigma^{2}$ vi) $e$ is normal. The Variables $X, Y$ and $e$ is already defined in section 1.

It has been recognized, however, that in numerous applications $X$ might be stochastic and $e$ might not be normal. This may give rise to three problems (a) $X$ is nonstochastic and $e$ is nonnormal, (b) $X$ is stochastic and $e$ is normal, and (c) $X$ is stochastic and $e$ is nonnormal.

The basic problem of the stochastic regressor in General Linear Model (GLM) is that the least square estimators may not be unbiased because when taking the expectation of the random vector $b$ (OLS estimator), $\mathrm{E}[\tilde{b}]=\tilde{\beta}+\mathrm{E}\left[\left(X^{\prime} X\right)^{-1} X^{\prime} \tilde{e}\right]$ the second term $\mathrm{E}\left[\left(X^{\prime} X\right)^{-1} X^{\prime} \tilde{e}\right]$ may not vanish. It may happen 
because $\mathrm{E}\left[\left(X^{\prime} X\right)^{-1} X^{\prime} \tilde{e}\right]$ cannot be expressed as $\left(X^{\prime} X\right)^{-1} X^{\prime} E[\tilde{e}]$ as in the case of fixed regressor, nor, due to the stochastic nature of $X$ and $e$, can be expressed as $E\left[\left(X^{\prime} X\right)^{-1} X^{\prime}\right] E[\tilde{e}]$ unless $X$ and $e$ can be assumed to be independent.

If $X$ is stochastic, the conceivable consequences of OLS estimators can be * unbiased, efficient \& consistent * biased but consistent * biased but inconsistent. Also, the distribution of OLS estimators $\hat{\beta}$ depends on the distribution of $e$ and $X$. In such cases statistical inference become more difficult. Appropriateness of OLS depends on the stochastic dependencies between the matrix $X$ and the error vector $e$. Three dependency structures are defined, in the first case error and regressor are stochastically independent, secondly, error term and regressor are contemporary uncorrelated and thirdly error term and regressor are contemporary correlated. In first two cases, OLS estimators are feasible since they are consistent. In the third case, application of alternative estimation procedures is needed since OLS estimators are biased and inconsistent. Instrumental Variable Regression can be applied in such cases.

Though Ehrenberg (1963) suggested that regression of stochastic regressor variables is useless and Kerridge (1967) suggested that it may be useful if the limitations are understood. But, Later on other methods were developed such as Modified Maximum Likelihood Estimator, more efficient than traditional OLS method (Islam and Tiku, 2010).

\section{A Bird eye view on works of Stochastic Regressors}

There is a huge literature related to stochastic regressors in various aspects. The basic assumption of classical linear regression model is that the values of the explanatory variables are fixed in repeated sampling, Hooper and Zellner (1961) treated the error of prediction of a multiple regression equation with constant regressor variable. Kerridge (1967) investigated the same but assumed that the regressor variables are drawn from a multivariate normal population. The k-dimensional vectors $X_{1}$, $X_{2}, \ldots, X_{n}$ are given as a random sample from the distribution $\mathrm{N}(\Lambda, \Sigma)$. Corresponding to this vector $Y_{1}, Y_{2}, \ldots, Y_{n}$ was observed. The error of prediction was expressed as the ratio of a normally distributed random variable and the square root of an independent beta-variate. It was realized that though such regression has limitations if the number of regressor variables is large, it may also be useful if the limitations were well understood.

In hypothesis testing on the coefficients of a linear regression model with stochastic regressors, $t$ and $\mathrm{F}$ test are applicable if the regressors are statistically independent of the disturbances. It was explained that there are also cases in which economic hypothesis can be stated in terms of the independence of stochastic regressors and disturbances. Wu (1973) examined four alternative tests of independence. He also examined two finite sample tests and two alternative asymptotic tests, along with power functions of them. It was shown that the entire tests were consistent. Here two linear models were considered where the disturbances are distributed as multivariate normal with mean zero and covariance matrix $\Sigma=\sigma_{11}\left(\begin{array}{c}\delta^{\prime} \\ \Sigma_{22}\end{array}\right.$. In $)$ 1973, Wu only presented four alternative tests of independence between stochastic regressors and disturbances in a linear regression model and shown that all the tests were consistent. Wu (1974), determined analytically the set of parameters that enters the power functions of the four tests and then compared the power functions of these tests using sampling experiment. Here also he considered two linear models where each row of the disturbances is independently distributed as multivariate normal with mean zero and covariance 
matrix $\quad \Sigma=\left(\begin{array}{cc}\sigma_{11} & \delta^{\prime} \\ \sigma & \Sigma_{22}\end{array}\right)$. This test is proposed for those problems in which the statistical decision making can be formulated as a choice between two alternative hypothesis of whether the regressors and disturbances are independent. He gave four examples of its applications in econometrics including a test of the permanent income hypothesis.

Narula (1971) presented that if prediction is the main objective, the mean square error of the predicted response from the observed response would seem to be a reasonable criterion, since it takes into account bias and variability simultaneously. Narula (1974), considered two approaches to improve the predictive mean square error of the predicted response when predictor variables are stochastic and follow a multivariate normal distribution. The first technique, the subset approach used only a subset of the available predictor variables to predict the response. To select the subset a decision rule was given. In the second method, the lambda approach, the regression coefficients were scaled down by a suitable constant. He then suggested an estimator of the constant. The two methods were then compared.

Wu (1973, 1974), Revankar and Hartley (1973), Revankar (1978), and Hwang (1978) have proposed test of independence between stochastic regressors and disturbance term in a single regression equation model. The assumption of these tests was that all right hand side variables besides those under test are exogenous or at least uncorrelated with the disturbance term. Hwang (1980) proposed the likelihood ratio test of independence of a subset of stochastic endogenous variables and the disturbance term. When the equation is over identified, however, the asymptotic null distribution of the likelihood ratio test is different from that of the full set likelihood ratio test. So, when the equation is just identified, the likelihood ratio test is equivalent to the Revankar-Hartley test (1973), Wu's T2 test, and Revankar's NT test (1978). When the equation is over identified, however, the asymptotic null distribution of the likelihood ratio test is different from that of the Revankar-Hartley test (1973), mainly because this does not consider the over identifying restrictions while the likelihood ratio test does. The subset likelihood ratio test is derived which is based on the ratio of two characteristic roots or equivalently based on the difference between the Anderson-Rubin test (1949) statistic of over identifying restrictions under the null and alternative hypothesis. Though the Anderson-Rubin test statistic of over identifying restrictions test is not widely used, can be used to test erogeneity of instrumental variables.

In many occurrences it is valuable to know whether stochastic regressors are independent of the disturbance terms in regression models. Reynolds (1982) developed the bayesian approach based on posterior odds ratios to considering the hypothesis of independence between stochastic regressors and disturbances and then compares it with the $\mathrm{Wu}$ tests. Here two simultaneous equations are considered, where the disturbances term are assumed independently and identically multivariate normally distributed with zero means and positive definite symmetric covariance matrices

$\Sigma=\left(\begin{array}{ll}\sigma_{11} & \sigma_{12}^{\prime} \\ \sigma_{12} & \Sigma_{22}\end{array}\right)$ This procedure provides an alternative procedure for testing hypothesis of erogeneity. The posterior probabilities of hypothesis that may be calculated from the posterior odds ratios also offer the intuitively appealing interpretation of reflecting the degree of belief that may be had in the hypothesis given the data. . He illustrated an example on an empirical model of the demand for health developed by Grossman (1972) and then provided a comparison with use of the Wu tests. The data used for considering this example are a sample of 816 observations of individuals in a 1970 national survey of health and health care conducted by the centre for Health Administration Studies (CHAS) 
at the University of Chicago. Based on information for both these methods under $\mathrm{H}_{\mathrm{o}}$ and $\mathrm{H}_{1}$ suggest that the data do not provide much basis for discriminating whether the consumption services of health are normal or inferior.

Strong consistency and asymptotic normality of least squares estimates in stochastic regression models were established under certain weak assumptions on the stochastic regressors and errors. Lai and Wei (1982) discussed about the application of the least square estimates in Stochastic Regression Models to identification and controls of dynamic systems. A multiple regression model is considered here.

Kinal and Lahiri (1983) analyzed the specification error problem in the context of a linear regression model with stochastic explanatory variables. This is more important for econometrics and sociological application since the researcher cannot set the values of the explanatory variables at predetermined levels such that they can be treated as fixed in repeated samples. The exact distribution of the omitted variable (OV) estimator is derived in the context of regression models with stochastic explanatory variables. Stochastic regressors present the problem of larger variance for the $\mathrm{OV}$ estimator than in the non-stochastic case, and derived conditions under which omission may be better under a minimum mean square error criterion. The error-in-variables problem can be interpreted as a specification error problem with stochastic regressors, and the issue of MSE dominance of the proxy-variable estimator over the OV estimator is considered. It may be optimal to introduce some measurement error, even in situations where all variables are correctly measured.

Potscher (1989) gave strong consistency results for model selection procedures based on variants of Akaike's information Criteria for general nonstationary stochastic linear regression models where the errors constitute a not necessary stationary martingale difference sequence using results of Lai and Wei $(1982,1983,1985)$. The results are then applied to yield strong consistency results for order estimation in nonstationary autoregressive models.

Lai (1994) established asymptotic properties of nonlinear least square estimates in stochastic regression models. Stochastic regression models, where the random disturbances form a martingale difference sequence with respect to an increasing sequence of $\sigma$-fields $\left\{\mathcal{G}_{i}\right\}$ and $f_{i}$ is a random $\mathcal{G}_{i-1}$ measurable function of an unknown parameter $\theta$, and cover a broad range of nonlinear (and linear) time series stochastic process models. In the linear case they reduced to known results on the linear least squares estimate with stochastic measurable regressors.

$\mathrm{Hu}$ (1997) used posterior covariance matrices to study the strong consistency of Bayes estimators in stochastic regression models under various assumptions on the stochastic regressors. The random errors were assumed to form a martingale difference sequence. Several results were obtained using a recursion satisfied by the sequence of posterior covariance matrices. These results suggested that the posterior covariance matrix is a useful tool in studying strong consistency problems in stochastic regression models. Three examples from sequential design and adaptive control are discussed.

In numerous situations, a bivariate random vector $(X, Y)$ is such that $Y$ depends on $X$ but not so much the other way round. Vaughan and Tiku (2000) assumed the distribution of $X$ to be the extreme value distribution and the conditional distribution of $Y$ to be normal. Then they derived the MML (modified maximum likelihood) estimators of the five parameters and showed that they are highly efficient. They also developed hypothesis testing procedures.

Akkaya and Tiku (2001) considered two distributions, the gamma (chi-square) with support $(0, \infty)$ and generalized logistic with support $(-\infty, \infty)$ for illustration. They derived MML (modified maximum likelihood) estimators of the parameters in the autoregressive model $y_{t}-\varphi y_{t-1}=\mu+\delta\left(x_{t}-\varphi x_{t-1}\right)+a_{t},(1$ 
$\leq t \leq n)$ and showed that they are asymptotically fully efficient for all values of $\varphi$. Their properties for small sample sizes are studied and showed that they are, like the LS (least squares) estimators, almost unbiased for all values of $\varphi$ but are considerably more efficient. They also developed procedures for testing the two important null hypotheses: (i) $\delta=0$, and (ii) $\varphi=0$. Testing $\varphi=1$ (the unit root problem) is of enormous practical interest but is considerably more difficult because the variance of $y_{t}$ is now infinite.

Magdalinos and kandilorou (2001) encompasses the problems of testing for structural restrictions and for valid orthogonality conditions into a single-equation framework in which the familiar specification techniques of the linear regression model can be applied. Identifying the endogenous variables that induce "simultaneous equation bias" will improve the efficiency of estimation, since only those variables need to be instrumented.

As in the linear regression model the regressors are traditionally assumed to be non stochastic, likewise it is often assumed that the random error is normally distributed. In numerous situations when dependent variable measures life times or reaction times, error term has skew distribution. Islam et al. (2001) considered two families of skew distributions, firstly Weibull with support IR: (0, $\infty)$ on the real line and secondly generalized logistic with support IR: $(-\infty, \infty)$. Then they derived Modified maximum likelihood estimators and showed that these estimators are efficient and robust. Then simulations are done for the means and variances for several values of $b$ (parameter) to compare the efficiencies of the MML estimators with the LS estimators. The biases in both the MML and the LS estimators were found to be negligible.

Tiku et al. (2001) patronized the salient features of a family of short-tailed symmetric distribution, and introduced the mechanisms for creating inliers. They assumed the error distribution to be one of this family, the methodology of modified likelihood is used to derive MML estimators of parameters in a linear regression model. The estimators are efficient and are the first to achieve robustness to inliers. And the design variable $x_{i}$ are generated from a uniform $(0,1)$ distribution and kept common for all $\mathrm{N}$ generated random samples. Then they further extended the case to long-tailed symmetric distributions and showed that the MML estimators have excellent efficiency and robustness properties. In particular, it can be said that they are robust to outliers.

In Regression Models, like Regressors, the errors are also traditionally assumed to be normally distributed. But in more recent years, there has been a realization that nonnormal distributions are more prevalent in practice. Islam and Tiku (2005) considered several nonnormal distributions for the random error $e$ of the equation $Y_{i}=\theta_{0}+\theta_{1} X_{i}+e_{i},(1 \leq \mathrm{i} \leq \mathrm{n})$, both symmetric and skew. Then they obtained the modified maximum likelihood estimators (MMLEs) of $\theta_{0}, \theta_{1}, \sigma$ and compared them with the least square estimators (LSEs) and Huber M estimators (1981). They showed that for plausible deviations from an assumed distribution, the MMLEs are more efficient and much easier to compute.

In many statistical applications, the deviations from normality are very common where usually the normality assumption invoked. Therefore there has been enormous practical interest to develop statistical procedures which are robust to plausible deviations from an assumed distribution particularly normal. As not much is known about how to obtain robust parametric estimators for short tailed distributions, so Akkaya and Tiku (2005) tried to develop such robust estimators by censoring a fixed proportion of ordered statistics in the middle of the random samples. They derived MMLE and showed that MMLE are explicit, and efficient and robust under short-tailedness and inliers. 
Sazak et al. (2006) considered situations when both $X$ and $e$ has non-normal distributions, i.e. when both the marginal and the conditional distributions are skew. Particularly generalized logistic distribution is taken for illustration for $X$ and $e$ with shape parameter $b_{1}(>0)$ and $b_{2}(>0)$. Then Modified Maximum Likelihood Estimation (MMLE) is used for parameter estimation which yields estimators which are asymptotically efficient. For small n, the MMLE are known to be essentially as efficient as the MLE (Maximum likelihood estimators) and the two are numerically very close to one another.

In literature, the risk factor $X$ in binary regression has been treated as a non-stochastic variable. However, in most situations, $X$ is stochastic. Oral (2006) presented solutions applicable to such situations where there are only four independent parameters. Here, $h(x)$ denotes the probability density function of $X$ and two cases are considered. In the first case $h(x)$ is skewed, e.g., Generalized Logistic (GL), whereas in the second case $h(x)$ is symmetric, e.g., scaled Student $t$. To study efficiency and robustness properties, MMLE is derived and showed that treating $X$ as nonstochastic results in loss of efficiency if $X$ is in fact stochastic. In particular, they showed that the traditional estimators based only on the conditional likelihood LYIX with the observations $x_{i}$ replaced by $\left(x_{i}-\bar{x}\right) / s, 1 \leq \mathrm{i} \leq \mathrm{n}$, give inefficient estimators unless $\mathrm{h}(\mathrm{x})$ is normal $N\left(0, \sigma^{2}\right)$.

Tiku and Akkaya (2010) derived Modified Maximum Likelihood Estimators of the parameters in a second order polynomial regression model. Modified Maximum Likelihood Estimators (MMLE) and Least Square Estimators (LSE) of the parameters in the second order polynomial regression model are obtained. Both are invariant to location and scale of the design variables and the former are considerably more efficient and, for any non-normal distribution, the relative efficiencies of LSEs are the same (almost) and less than 1 for all designs considered. They illustrated with three real life examples.

Islam and Tiku (2010) tried to developed estimators of parameters when there are $q \geq 2$ stochastic design variables in the multiple linear regression models, which is more general and challenging situations. It may be noted that treating $X_{j,}(1 \leq j \leq q)$ as non-stochastic when, in fact, they are stochastic can unknowingly yield estimators which have considerable bias and are inefficient. Both Modified Maximum Likelihood Estimation (MMLE) and Least Square Estimator (LSE) Method are applied for parameter estimation. A comprehensive Monte Carlo simulation study using $[100,000 / n]$ Monte Carlo runs is carried out to evaluate the bias and efficiency of the LSE and the MMLE. Both the methods have negligible bias. However, MMLE method is considerably more efficient.

Tiku and Akkaya (2010) derived Modified maximum likelihood Estimators (MMLEs) and Least Square Estimators (LSEs) of the parameters in a second order polynomial regression model where the random error term are assumed to follow Long -Tailed Symmetric Distributions (LTD), Generalized Logistic, Short-Tailed Symmetric Distribution. They illustrated and compared their findings with four real life examples and found that MMLE are more efficient and robust than the LSEs.

\section{Conclusions}

In the world of econometrics, regression analysis plays an important role. Linear Regression model is widely used in biological, behavioral and social sciences to describe possible relationships between variables. A large number of procedures have been developed for parameter estimation and inference in linear regression model with respect to distributions, theoretical assumptions for the 
validity of the test. The least squares principle can be applied over the regression model to estimate the parameters and for Best Linear Unbiased Estimators (BLUE) it must satisfy some basic assumptions. Maximum Likelihood Estimation can be performed when the distributions of the error terms is known to belong to a certain parametric family of probability distributions. However, when the parametric family is normal distributions with mean 0 and variance $\sigma^{2}$, the resulting estimate is identical to the Ordinary Least Squares Estimation. Violations of these basic assumptions lead to serious consequences of the model development. One of the important assumptions is that the regressors are fixed in repeated samples. However, in many cases particularly in social sciences the assumption of fixed regressor is not always tenable. It may be stochastic in nature. The practical strategy to follow the assumption of fixed regressor is rationale though the variable may be intrinsically stochastic. The literature survey reveals that the instance of stochastic regressor is truly a fascinating area where the regressor may follow some traditional distributions. The regressor may follow other distribution(s) besides normality. In cases of non-normality, the traditional estimation method is in general problematic. For instance, the likelihood equations have no explicit solutions and solving them by iteration can be problematic. To attenuate these difficulties, Method of Modified Maximum Likelihood (MMLE) is used, introduced by Tiku $(1967,1968,1980)$ and Tiku and Suresh (1992), Islam et al. (2001) and Tiku et al. (2001) developed MMLE for X nonstochastic and e nonnormal and considered the following families of distributions: Weibull, Generalized logistic, Student's $t$, and short-tailed distributions. There is a huge literature related to stochastic regressors in various aspects. So, based on literature search a historical perspective on some of the works related to stochastic regressors has been critically discussed here which fulfills the objective of this paper. However, after 2010, so far our knowledge goes no significant attempt has been made by the researchers in this area.

\section{References}

(Akkaya and Tiku 2001) Akkaya, A., Tiku M.L. (2001) Estimating parameters in autoregressive models in non-normal situations: Asymmetric innovations. Communication in StatisticsTheory and Methods 30(3):517-536.

(Akkaya and Tiku 2005) Akkaya A.D., Tiku M.L.2005. Robust estimation and Hypothesis Testing under Short-Tailedness and Inliers. Test 14(1): 129-150.

(Bowden and Turkington 1981) Bowden R.J., Turkington D.A. 1981. A Comparative Study of Instrumental Estimators for Nonlinear Simultaneous Models. Journal of the American Statistical Association 76 (3): 988-995.

(Chow 1984) Chow, G.C. (1984) Econometrics. McGRAW-HILL Education (ISE Editions). Economic Series.

(Davidson and Mackinnon 1999) Davidson, R. and Mackinnon, J.G. (1999) Econometric Theory and Methods. Oxford University Press

(Ehrenberg 1963) Ehrenberg, A.S.C. (1963) Bivariate Regression Analysis is useless. Journal of the Royal Statistical Society (Applied. Statistics) 12:161-179. 
(Gorman and Toman 1966) Gorman, T.W. and Toman, R.J. (1966) Selection of variables for fitting equations to data. Technometrics 8: 27-51.

(Greene 2006) Greene, W.H. (2006) Econometric Analysis. Dorling Kindersley Pvt. Ltd.

(Grossman 1972) Grossman M (1972) The Demand for Health: A Theoretical and Empirical Investigation (New York: National Bureau of Economic Research). Columbia University Press.

(Hartley 1973) Hartley, M.J. (1973) An Independence Test and Conditional Unbiased Predictions in the Context of Simultaneous Equation Systems. International Economic Review 14: 625-631.

(Hooper and Zellner 1961) Hooper, J.W. and Zellner, A. (1961) The error of forecast for multivariate regression models. Econometrica 29: 544-555.

(Hu 1997) Hu, I. (1997) Strong Consistency in Stochastic Regression Models via Posterior Covariance Matrices. Biometrika 84(3): 744-749.

(Huber 1981) Huber, P.J. (1981) Robust Statistics. New York: John Wiley \& Sons.

(Hwang 1978) Hwang, H. (1978) Further Tests of Independence. Mimeo.

(Hwang 1980) Hwang, H. (1980) Test of Independence between a subset of Stochastic Regressors and Disturbances. International Economic Review 21(3):749-760.

(Islam et al. 2001) Islam, M.Q., Tiku, M.L. and Yildirim, F. (2001) Nonnormal Regression. I. Skew distribution. Communications in Statistics -Theory and Methods 30(6): 993-1020.

(Islam and Tiku 2005) Islam, M.Q., Tiku, M.L. (2005) Multiple Linear Regression Model Under Nonnormality. Communications in Statistics -Theory and Methods 33(10): 2443-2467.

(Islam and Tiku 2010) Islam, M.Q. and Tiku, M.L. (2010) Multiple linear regression model with stochastic design variables. Journal of Applied Statistics 37(6):923-943.

(Judge et al. 1988) Judge, G.G., Hill, R.C., Griffiths, W.E., Lutkepohl, H. and Lee, T. (1988) Introduction to the theory and practices of econometrics. John Wiley and Sons.

(Kerridge 1967) Kerridge, D. (1967) Errors of prediction in multiple regressions with stochastic regressor variables. Technometrics 9: 309- 311.

(Kinal and Lahiri 1983) Kinal, T. and Lahiri, K, (1983) Specification Error Analysis with Stochastic Regressors. Econometrica 51(4): 1209-1219.

(Lai 1994) Lai, T. (1994) Asymptotic Properties of Nonlinear Least Squares Estimates in Stochastic Regression Models. The Annals of Statistics 22(4): 1917-1930.

(Lai and Wei 1982) Lai, T.L., Wei, C.Z. (1982) Least Squares Estimates in Stochastic Regression Models with Applications to Identification and Control of Dynamic Systems. The Annals of Statistics 10(1): 154-166.

(Lai and Wei 1983) Lai, T.L., Wei, C.Z. (1983) Least Asymptotic properties of general autoregressive models and strong consistency of least-squares estimates of their parameters. Journal of Multivariate Analysis 13(1): 1-23.

(Lai and Wei 1985) Lai, T.L., Wei, C.Z. (1985) Least Asymptotic properties of multivariate weighted sums with application to stochastic regression in linear dynamic systems. In P.R. Krishnaiah (ed.) Multivariate Analysis VI: 375-393. 
(Maddala 2010) Maddala, G.S. (2010) Introduction to Econometrics. Wiley India Pvt. Ltd.

(Magdalinos and kandilorou 2001) Magdalinos, M. and kandilorou, H. (2001) Specification Analysis in Equations with Stochastic Regressors. Journal of Bussiness \& Economic Statistics 19(2): 226232.

(Malinvaud 1980) Malinvaud, E. (1980) Statistical Methods of Econometrics. North Holland Publishing Company. Amsterdam, New York, Oxford.

(Nachane 2006) Nachane, D.M. (2006) Econometrics. Oxford University Press.

(Narula 1971) Narula, S.C. (1971) Least squares regression with mean square error criterion. PhD. Dissertation. University of Iowa.

(Narula 1974) Narula, S.C. (1974) Predictive mean Square Error and Stochastic Regressor Variables. Journal of the Royal Statistical Society. Series C(applied Statistics) 23(1): 11-17.

(Oral 2006) Oral, E. (2006) Binary Regression with Stochastic Covariates. Communications in Statistics-Theory and Methods 35(8):1429-1447.

(Potcher 1989) Potcher, B.M. (1989) Model Selection under Nonstationarity: Autoregressive Models and Stochastic Linear Regression Models. The Annals of Statistics 17(3): 1257-74.

(Ramanathan 1993) Ramanathan, R. (1993) Statistical Methods in Econometrics. Academic Press.

(Revankar 1978) Revankar, N.S. (1978) Asymptotic Relative Efficiency Analysis of Certain Tests of Independence in Structural Systems. International Economic Review 19: 165-179.

(Reynolds 1982) Reynolds, R.A. (1982) Posterior Odds for the Hypothesis of Independence between Stochastic Regressors and Disturbances. International Economic Review 23(2): 479-490.

(Sazak et al. 2006) Sazak HS, Tiku ML, Islam MQ (2006) Regression Analysis with a Stochastic Design Variable, International Statistical Review 74(1):77-88.

(Smith 1984) Smith, R.J. (1984) A Note on likelihood Ratio Tests for the Independence between a Subset of Stochastic Regressors and Disturbances 25(1): 263-269.

(Stigler 1954) Stigler, G.J. (1954) The Early History of Empirical Studies of Consumer Behaviour. The Journal of Political economy 62(2):95-113.

(Tiku 1967) Tiku, M.L. (1967) Estimating the mean and standard deviation from a censored normal sample. Biometrika 54: 155-165.

(Tiku 1967) Tiku, M.L. (1968) Estimating the parameters of normal and logistic distributions from censored samples. Australian Journal of Statistics 10: 64-74.

(Tiku 1967) Tiku, M.L. (1980) Robustness of MML estimators based on censored samples and robust test statistics. Journal of Statistical Planning and Inference 4: 123-143.

(Tiku and Suresh 1992) Tiku, M.L. and Suresh, R.P. (1992) A new method of estimation for location and scale parameters. Journal of Statistical Planning and Inference 30: 281-292.

(Tiku et al. 2001) Tiku, M.L., Islam, M.Q. and Selcuk, A.S. (2001) Nonnormal Regression. II. Symmetric Distributions. Communications in Statistics - Theory and Methods 30(6):1021-1045.

(Tiku and Akkaya 2010) Tiku, M.L., Akkaya, A.D. (2010) Estimation in Multifactor Polynomial Regression under Non-Normality. Pakistan Journal of Statistics 26(1):49-68. 
(Vaughan and Tiku 2000) Vaughan, D.C. and Tiku, M.L. (2000) Estimation and Hypothesis Testing for a Nonnormal Bivariate Distribution with Applications. Mathematical and Computer Modeling 32: 53-67.

(Wu 1973) Wu De-Min (1973) Alternative Tests of Independence between Stochastic Regressors and Disturbances. Econometrica 41(4): 733-750.

(Wu 1974) Wu De-Min (1974) Alternative Tests of Independence between Stochastic Regressors and Disturbances: Finite Sample Results. Econometrica 42(3):529-546. 